\title{
Editorial
}

\section{Obligatory information on patient-oriented end point outcome}

\author{
P. Riis \\ Council of Europe, Working Party on Biomedical Research, D - 2900 Hellerup, Denmark
}

Up to 50 years ago personal experience was still the major ingredient of a clinician's professional competence. Even with the reading of medical journals and attendance of scientific meetings the information collected from contact with patients was the esteemed basis for judging a clinician's skill. In the perspective of the long history of medicine it is strange that despite isolated attempts made by clinical thinkers to analyse clinical decision making and its logical components $[1,2,3]$, it took centuries before Fibiger in 1898 [2] and Bradford-Hill in 1948 planned and carried through the first proper randomised controlled clinical trials (RCTs) and before this was followed up with the formulation of a set of scientific tools, named methodology [4].

The basic principles. Familiarity with clinical scientific methodology presupposes an understanding of the two underlying axioms: everything moves in clinical medicine and all judgements rest on comparisons.

That biological variation is inherent in clinical medicine is illustrated by that found within diagnostic groups, i.e. patient-to-patient variation seen in daily contact with patients and that a similar variation is ubiquitous within individual patients over time. A number of technical procedures have been derived from the two axioms aimed at controlling these two main sources of variation to reduce variation to the one caused by the clinical intervention tested: randomization, blinding and correction for extreme, non-intervention derived variation, i.e. statistics. Refined clinical scientific methodology today forms the basis of so-called 'evidence-based medicine'. The application of modern methodology serves two purposes: to plan scientific protocols and for clinicians to use the results of RCTs critically in making decisions for individual patients.

Which end points should be chosen? This is the starting point of the divergence between the stand taken by Vijan et al. [5] and Richter et al. [6]. Scientists do not invariably plan their trials in a way that serves the interests of patients and their clinicians. Clinical research protocols are not always directed at relevant clinical end points but often have the sole aim of explaining intermediate end points, for instance intervention-elicited changes in key variables such as blood glucose, blood pressure, or reduced concentrations of inflammatory mediators.

Sometimes such intermediary end points are important as pathophysiological steps towards patientoriented end points but often the investigative policy stops here. In so doing it leaves the illusion that clinicians will be able to apply the results directly to patients and forgets that patients rightfully are not interested in changed intermediate variables if they do not with reasonable safety reflect changes in symptom rating, possibility of cure or the extreme variation, life versus death.

Apart from the lack of methodological insight in planning trials, thereby underestimating the control mechanisms derived from the two axioms, the motives behind the choice of intermediary end points can be more complex. Both scientists and drug firms sometimes prefer fast results which do not marry up with the complexity and duration of the application of patient-oriented end points. For instance a trial comparing a new drug, or an analogue of an existing one, reaching a statistically significant result with few resources and little time spent can produce scientifically relevant (but not always clinically relevant) results that sometimes influence both clinicians prescribing drugs and academic leaders evaluating the scientific merits of applicants.

What should be done? Preventive measures should start by convincing research ethics committees to define their minimum level of acceptable methodology before they ratify protocols. A policy which would surely prevent the acceptance of, as an exaggerated 
example, a protocol to measure a new drug's effect solely on body temperature reduction within 2 days in pneumonia patients, should be extended to prevent acceptance of other types of non-relevant or insufficient end points.

When research ethics committees do find an intermediary end point acceptable as a stepwise and pathophysiological way to later therapeutical improvements the next preventive guardians ought to be editors and referees. They should be alerted to methodological quality and its dependence on the choice of end points. Their responsibility should be to ensure that any manuscript for publication correctly states only a limited conclusion and not one transferring changes in an intermediate variable to patient-oriented end points where this was never a part of the trial protocol.

There are still two technical obstacles preventing clinical scientists from embarking on projects comparing different drug therapies, individually or in combinations, in large-scale studies, based on longterm patient-oriented end point outcomes. One is the right in some countries of drug producers to veto planned scientific trials including the firm's registered drug unless the national drug agency grant an exception. The other is the economic resources needed to run such trials. Often the drug industry is reluctant to, or outright negative in, supporting large scale comparative studies. Independent public funds are much needed as alternative sources of support, especially when the disease is a major health problem for society.

Even if controlled trials as part of evidence-based medicine are planned and carried through according to the best methodological standards with control groups, randomization, blinding, patient-oriented end point outcomes, corrections for type one and type two errors etc., the results still need supplementary evidence from different sources to be applicable and decisive for the individual patient. Evidencebased medicine in the form of the classical paradigm, the randomized controlled trial, is necessary but alone is not sufficient for good clinical decision making.

Which supplements are needed? Firstly, the characterization of the sample groups of patients included in research protocols needs to be improved. The clinician who uses the results of the research will then with greater safety be able to judge whether his or her patient was represented in principle in the trial sample still with the built-in bias that results are given as ranges, averages or means and confidence limits, which always involves a substantial loss of detail. Secondly, patient-oriented end point outcomes should be accompanied by obligatory information on a given intervention's economic cost at the time of publication compared with possible alternatives. Thirdly, the results of RCTs should be applied in single patient cases also taking account of the hopes and preferences of patients, the economic accessibility of a given intervention, evidence from observational and epidemiological studies on the influence of lifestyles on spontaneous outcomes or as interactions with other treatments etc.

The pedagogic spin-off of RCTs. Controlled clinical studies have had a revolutionary impact on clinical research and on the way clinicians read the professional literature, register and file their personal experience and deal with other sources of evidence from observational studies. Applying the two axioms, mentioned above, outside the randomized, double-blind drug trial has extended the spectrum of qualified evidence necessary for competent clinicians to exploit in concrete patient situations. As a supplement to scientific overview articles, editorials, Cochrane analyses etc. this secondary educational impact cannot be overemphasized.

The cultic sides of the RCT. Those of us who 40-50 years ago experienced the conceptual breakthrough of the controlled clinical study and have even worked hard to spread the good tidings in writing, editing and lecturing are aware that the RCT has obtained an almost cultic position. The very positive aspects of this development are welcomed but there are also minor side effects. If the RCT paradigm is maintained too rigidly it will tend to characterize all evidence sources outside the paradigm (qualitative studies, observational studies, epidemiological studies etc.) as non-scientific and sometimes even call them generally misleading. If instead the basic principles and tools of the RCT are applied to other evidence sources with, acceptance of and corrections for, the omnipresent bias-sources, the result will be a wider lift in the quality of clinical decision making, instead of referring to areas outside the rigid paradigm as guessing, believing and whatever else was formerly a part of the falsely awed "art of medicine" in individual clinical decision making.

\section{References}

1. Lind J (1753) A treatise of the scurvy. Edinburgh

2. Fibiger J (1898) Om serumbehandling af difteri. Hospitalstid 6: 309-325 and 338-350

3. Vandenbroucke JP (1987) A short note on the history of the randomized controlled trial. J Chron Dis 40: 985-987

4. Medical Research Council (1948) Streptomycin treatment of pulmonary tuberculosis. BMJ 2: 769-782

5. Vijan S, Kent DM, Hayward RA (1999) Are randomized controlled trials sufficient evidence to guide clinical practice in Type II (non-insulin-dependent) diabetes mellitus? Diabetologia (in press)

6. Richter B, Berger M (1999) Randomized controlled trials remain fundamental to clinical decision making in Type II diabetes mellitus. Diabetologia (in press) 